\title{
Optimal Control and Cost-Effectiveness Analysis of HIV Model with Educational Campaigns and Therapy
}

\author{
Marsudi*, Noor Hidayat and Ratno Bagus Edy Wibowo \\ Department of Mathematics, University of Brawijaya \\ Malang 65145, Indonesia \\ *Correspondence author: marsudi61@ub.ac.id
}

Article history

Received: 15 November 2019

Received in revised form: 26 November 2019

Accepted: 23 December 2019

Published online: 31 December 2019

\begin{abstract}
In this paper, we present a deterministic model for the transmission dynamics of HIV, in which educational campaigns and therapy are both important for disease management. We propose and analyze an optimal control problem to investigate the effectiveness and cost-effectiveness of three control measures (educational campaigns, therapy on infected individuals in the asymptomatic stage, and therapy on infected individuals in the pre-AIDS class). We formulate the appropriate optimal control problem and investigate the necessary conditions for disease control in order to determine the role of asymptomatic infection, pre-AIDS, and full-blown AIDS in the spread of HIV. Pontryagin's Maximum Principle was employed to derive the necessary conditions for the existence of optimal control. The fourth-order Runge-Kutta forward-backwards sweep numerical approximation method was used to solve the optimal control system. The Incremental Cost-Effectiveness Ratio (ICER) was calculated to investigate the costeffectiveness of all possible combinations of the three control measures. Using costeffectiveness analysis, we showed that control of therapy on pre-AIDS and a combination of control of educational campaigns and therapy on pre-AIDS provides the most costeffective strategy to control the disease.
\end{abstract}

Keywords Human Immunodeficiency virus (HIV); optimal control measure; Hamiltonian; cost-effective intervention; numerical simulations.

Mathematics Subject Classification 49J15, 93C15, 93C95

\section{Introduction}

The national (government) response for the HIV/AIDS epidemic focuses on a variety of comprehensive policies and programs. One of the pillars that are key to the success of HIV/AIDS prevention is care, support, and treatment with the provision of antiretroviral therapy and prevention through educational campaigns of condoms use for groups at risk of HIV/AIDS transmission. At present, the development of the effectiveness of condoms use 
campaign programs and antiretroviral therapy is inadequate, even though program coverage has increased.

Mathematical modeling plays an important role in understanding the dynamics of epidemics and describing epidemiological problems[1, 2, 3]. Mukandavire et al. [4] examined the problem of the spread of HIV/AIDS in association with public health education interventions. Furthermore, some studies [5, 6, 4] have included educational campaigns. Treatment (therapy) is important to reduce the spread of HIV/AIDS $[7,5,4,6,8,9]$. Other studies are related to the modeling of the effect of risky sexual behavior on the spread of HIV/AIDS [5, 10].

Optimization and optimal control problems have received much attention from researchers $[11,12,5,13,14,15,16,4,6,10,17,18]$. Okosun et al. [17] presented optimal control strategies and cost-effectiveness analysis of a malaria model. Furthermore, one study [17] presented the impact of optimal control on the treatment of HIV/AIDS and the screening of unaware infected individuals. From this study, it was concluded that a combination of three intervention strategies was more effective than without intervention. If the costs and health outcomes of these interventions are available, then the cost-effectiveness between one strategy and another can be compared. To achieve this goal, it is necessary to calculate the incremental cost-effectiveness ratio (ICER), which is the ratio between the difference in costs and health outcomes from the intervention.Motivated by the results of Okosun et al. [17], in this paper we propose to improve the work by Marsudi et al. [6] by including the aspect of antiretroviral therapy on full-blown AIDS in a homogeneous population. It is well known that antiretroviral therapy on full-blown AIDS may also play a major role in the transmission dynamics of the disease.

The following is the organization of the paper. In Section 2, we present the model formulation. Section 3 presents the analysis of optimal control. Section 4 presents the numerical simulations of the model and the cost-effectiveness analysis. Finally, we end with a conclusion in Section 5.

\section{Model Formulation}

In this paper, the model refers to the model proposed in Marsudi et al. [6] by adding control of antiretroviral therapy on full-blown AIDS. The model considers six disjoint classes: $S(t)$ represents susceptible individuals, $E(t)$ represents susceptible individuals who receive educational campaigns, $I(t)$ represents asymptomatic infected individuals, $P(t)$ represents symptomatic infected individuals or pre-AIDS individuals, $A(t)$ represents full-blown AIDS individuals, and $T(t)$ represents infected individuals who receive antiretroviral therapy, or treated individuals. The total population at any time $t$, denoted by $N(t)$, is the sum of individual populations in each class, such that $N(t)=S(t)+E(t)+I(t)+P(t)+T(t)+A(t)$.

The formulation of the model is based on the interactions among classes and the following assumptions:

1. Susceptible individuals can be infected through sexual contact with the two infected classes $I$ and $P$. It is assumed that the rates of contact of susceptible individuals with classes $I$ and $P$ are at different rates of $\beta_{1}$ and $\beta_{2}$ respectively, where $\beta_{2}<\beta_{1}$. We use standard incidence to model the disease transmission.

2. The rate of recruitment of susceptible individuals by birth or immigration is $\Lambda$. 
3. Susceptible individuals can be educated at a rate of $u_{1}$ and the effectiveness of the educational campaign is $\delta$.

4. Pre-AIDS and full-blown AIDS individuals are treated with therapy at successive rates of $u_{2}$ and $u_{3}$.

5. Only infected pre-AIDS individuals will become full-blown AIDS individuals at a rate of $\sigma_{2}$.

6. The progression rate from asymptomatic infection to pre-AIDS infection is $\sigma_{1}$.

7. The natural death of all classes is $\mu$. The disease-induced death rate of $T$ is $\alpha_{1}$ and the disease-induced death rate of $A$ is $\alpha_{2}$.

8. All parameters are assumed to be non-negative.

The HIV/AIDS model was developed with respect to time-dependent control variables (models with controls), which are control of educational campaigns on susceptible individuals $\left(u_{1}\right)$, control of antiretroviral therapy on pre-AIDS individuals $\left(u_{2}\right)$, and control of antiretroviral therapy on full-blown AIDS individuals $\left(u_{3}\right)$. The control functions $u_{1}, u_{2}$, and $u_{3}$, were defined at the closed interval $\left[0, T_{f}\right]$, where $0 \leq u_{i}(t) \leq 1, t \in\left[0, T_{f}\right], i=1,2,3$ and $T_{f}$ denotes the end time of controls.

Based on the above assumptions, we can formulate our model as the following deterministic system of non-linear differential equations:

$$
\begin{aligned}
& \frac{d S}{d t}=\Lambda-\beta S-u_{1} S-\mu S \\
& \frac{d E}{d t}=u_{1} S-(1-\delta) \beta E-\mu E \\
& \frac{d I}{d t}=\beta S+(1-\delta) \beta E-\left(\sigma_{1}+\mu\right) I \\
& \frac{d P}{d t}=\sigma_{1} I-\left(\sigma_{2}+u_{2}+\mu\right) P \\
& \frac{d T}{d t}=u_{2} P+u_{3} A-\left(\alpha_{2}+\mu\right) T \\
& \frac{d A}{d t}=\sigma_{2} P-\left(u_{3}+\alpha_{1}+\mu\right) A
\end{aligned}
$$

where we denote $\beta=\left(\beta_{1} I+\beta_{2} P\right) / N$ and $N=S+E+I+P+T+A$ with initial conditions

$$
S(0)=S_{0}, E(0)=E_{0}, I(0)=I_{0}, P(0)=P_{0}, T(0)=T_{0} \text {, and } A(0)=A_{0} .
$$

It is not difficult to verify that all feasible solutions of system (1) are bounded and enter the region

$$
\Omega=\left\{(S, E, I, P, T, A) \in R_{+}^{6} \mid N \leq \frac{\Lambda}{\mu}\right\} .
$$


Our goal is to minimize the number of cases in infected subpopulations $(I, P$ and $A)$ as well as to minimize the cost of control for educational campaigns $\left(u_{1}\right)$, therapy of pre-AIDS individuals $\left(u_{2}\right)$, and therapy of full-blown AIDS individuals $\left(u_{3}\right)$. The objective function $J$ is given by

$$
J\left(u_{1}, u_{2}, u_{3}\right)=\int_{0}^{T_{f}}\left[I+P+A+\frac{1}{2}\left(C_{1} u_{1}^{2}+C_{2} u_{2}^{2}+C_{3} u_{3}^{2}\right)\right] d t .
$$

The constants $C_{i} \geq 0(i=1,2,3)$ are weights of the relative costs of the associated controls $u_{1}, u_{2}$, and $u_{3}$, respectively. In other words, we seek an optimal control triple $\left(u_{1}^{*}, u_{2}^{*}, u_{3}^{*}\right)$ such that

$$
J\left(u_{1}^{*}, u_{2}^{*}, u_{3}^{*}\right)=\min \left\{J\left(u_{1}, u_{2}, u_{3}\right) \mid u_{1}, u_{2}, u_{3} \in U\right\}
$$

where $U=\left\{\left(u_{1}, u_{2}, u_{3}\right) \mid 0 \leq u_{i} \leq 1, i=1,2,3, \forall t \in\left[0, T_{f}\right]\right\}$ is the control set.

\section{Analysis of Optimal Control}

In this section, we analyze system (1) with its control functions $u_{1}, u_{2}$, and $u_{3}$ and theobjective functional equation (3). The optimal control triple $\left(u_{1}^{*}, u_{2}^{*}, u_{3}^{*}\right)$ must satisfy the necessary conditions that are formulated by Pontryagin's Maximum Principle [16, 20]. This principle converts Equations (1) and (3) into a problem of minimizing pointwise a Hamiltonian $\mathrm{H}$ with respect to the controls $\left(u_{1}, u_{2}, u_{3}\right)$. We formulate the Hamiltonian from the cost function of Equation (3) and the governing dynamics equation (1) to obtain the optimality conditions. The Hamiltonian function $H$ associated without problem is

$$
\begin{aligned}
H=I+ & P+A+\frac{1}{2}\left(C_{1} u_{1}^{2}+C_{2} u_{2}^{2}+C_{3} u_{3}^{2}\right)+\lambda_{s}\left[\Lambda-\frac{\left(\beta_{1} I+\beta_{2} P\right) S}{N}-u_{1} S-\mu S\right] \\
+ & \lambda_{E}\left[u_{1} S-\frac{(1-\delta)\left(\beta_{1} I+\beta_{2} P\right) E}{N}-\mu E\right] \\
+ & \lambda_{I}\left[\frac{\left(\beta_{1} I+\beta_{2} P\right) S}{N}+\frac{(1-\delta)\left(\beta_{1} I+\beta_{2} P\right) E}{N}-\left(\sigma_{1}+\mu\right) I\right] \\
& +\lambda_{P}\left[\sigma_{1} I-\left(\sigma_{2}+u_{2}+\mu\right) P\right]+\lambda_{T}\left[u_{2} P+u_{3} A-\left(\alpha_{2}+\mu\right) T\right] \\
& +\lambda_{A}\left[\sigma_{2} P-\left(\alpha_{1}+u_{3}+\mu\right) A\right] .
\end{aligned}
$$

with $\lambda=\left(\lambda_{S}, \lambda_{E}, \lambda_{I}, \lambda_{P}, \lambda_{T}, \lambda_{A}\right)$ being the adjoint vector related to the state variables $x=$ $(S, E, I, P, T, A)$. Assume that $(x, u)$ is an optimal solution of the optimal control problem (1)-(3). Then, there is a non-trivial vector function $\lambda=\left(\lambda_{S}, \lambda_{E}, \lambda_{I}, \lambda_{P}, \lambda_{T}, \lambda_{A}\right)$ such that

$$
\frac{d x}{d t}=\frac{\partial H}{\partial \lambda}, \quad \frac{\partial H}{\partial u}=0 \quad \frac{d \lambda}{d t}=-\frac{\partial H}{\partial x} .
$$

with the transversality condition

$$
\lambda_{j}\left(T_{f}\right)=0, \quad j=S, E, I, P, T, A .
$$

Hence, we obtain the following result:

Theorem 1 Let $\left(S^{*}, E^{*}, I^{*}, P^{*}, T^{*}, A^{*}\right)$ be optimal state solutions with associated optimal control variables $\left(u_{1}^{*}, u_{2}^{*}, u_{3}^{*}\right)$ for the optimal control problem (1)-(3) with given initial conditions 
$(S(0), E(0), I(0), P(0), T(0), A(0))$ and fixed final time $T_{\mathrm{f}}$. Then, there exist the adjoint variables $\lambda_{j}, j=S, E, I, P, T, A$ satisfying

$$
\begin{aligned}
\frac{d \lambda_{S}}{d t} & =\left(\lambda_{S}-\lambda_{I}\right)\left[\frac{\beta_{1} I+\beta_{2} P}{N}-\frac{\left(\beta_{1} I+\beta_{2} P\right) S}{N^{2}}\right]+\left(\lambda_{I}-\lambda_{E}\right)\left[\frac{(1-\delta)\left(\beta_{1} I+\beta_{2} P\right) E}{N^{2}}\right]+\left(\lambda_{S}-\lambda_{E}\right) u_{1}+\lambda_{S} \mu \\
\frac{d \lambda_{E}}{d t} & =\left(\lambda_{E}-\lambda_{I}\right)\left[\frac{(1-\delta)\left(\beta_{1} I+\beta_{2} P\right)}{N}-\frac{(1-\delta)\left(\beta_{1} I+\beta_{2} P\right) E}{N^{2}}\right]+\left(\lambda_{I}-\lambda_{S}\right)\left[\frac{\left(\beta_{1} I+\beta_{2} P\right) S}{N^{2}}\right]+\lambda_{E} \mu \\
\frac{d \lambda_{I}}{d t} & =-1+\left(\lambda_{S}-\lambda_{I}\right)\left[\frac{\beta_{1} S}{N}-\frac{\left(\beta_{1} I+\beta_{2} P\right) S}{N^{2}}\right]+\left(\lambda_{E}-\lambda_{I}\right)\left[\frac{(1-\delta) \beta_{1} S}{N}-\frac{(1-\delta)\left(\beta_{1} I+\beta_{2} P\right) E}{N^{2}}\right] \\
& +\left(\lambda_{I}-\lambda_{P}\right) \sigma_{1}+\lambda_{I} \mu \\
\frac{d \lambda_{P}}{d t} & =-1+\left(\lambda_{S}-\lambda_{I}\right)\left[\frac{\beta_{2} S}{N}-\frac{\left(\beta_{1} I+\beta_{2} P\right) S}{N^{2}}\right]+\left(\lambda_{E}-\lambda_{I}\right)\left[\frac{(1-\delta) \beta_{2} E}{N}-\frac{(1-\delta)\left(\beta_{1} I+\beta_{2} P\right) E}{N^{2}}\right] \\
& +\left(\lambda_{P}-\lambda_{A}\right) \sigma_{2}+\left(\lambda_{P}-\lambda_{T}\right) u_{2}+\lambda_{P} \mu \\
\frac{d \lambda_{T}}{d t} & =\left(\lambda_{I}-\lambda_{S}\right)\left[\frac{\left(\beta_{1} I+\beta_{2} P\right) S}{N^{2}}\right]+\left(\lambda_{I}-\lambda_{E}\right)\left[\frac{(1-\delta)\left(\beta_{1} I+\beta_{2} P\right) E}{N^{2}}\right]+\lambda_{T}\left(\alpha_{2}+\mu\right) \\
\frac{d \lambda_{A}}{d t} & =-1+\left(\lambda_{I}-\lambda_{S}\right)\left[\frac{\left(\beta_{1} I+\beta_{2} P\right) S}{N^{2}}\right]+\left(\lambda_{I}-\lambda_{E}\right)\left[\frac{(1-\delta)\left(\beta_{1} I+\beta_{2} P\right) E}{N^{2}}\right]+\left(\lambda_{A}-\lambda_{T}\right) u_{3}+\lambda_{A}\left(\alpha_{1}+\mu\right) .
\end{aligned}
$$

and with transversality condition (7).

Furthermore, when boundary conditions for $0 \leq u_{i} \leq 1, i=1,2,3$ are used in the control, the optimal control $\left(u_{1}^{*}, u_{2}^{*}, u_{3}^{*}\right)$ is obtained such that

$$
\begin{aligned}
& u_{1}^{*}=\min \left\{\max \left(0, \frac{\left(\lambda_{S}-\lambda_{E}\right) S^{*}}{C_{1}}\right), 1\right\}, \\
& u_{2}^{*}=\min \left\{\max \left(0, \frac{\left(\lambda_{P}-\lambda_{T}\right) P^{*}}{C_{2}}\right), 1\right\}, \\
& u_{3}^{*}=\min \left\{\max \left(0, \frac{\left(\lambda_{A}-\lambda_{T}\right) A^{*}}{C_{3}}\right), 1\right\} .
\end{aligned}
$$

Proof The existence of optimal control can be obtained using a result by Fleming and Rishel [13]. The adjoint equation is found by differentiating the Hamiltonian equation (5) with respect to state variables.

$$
\begin{aligned}
\frac{d \lambda_{S}}{d t}=-\frac{\partial H}{\partial S}, & \lambda_{S}\left(T_{f}\right)=0, & \frac{d \lambda_{P}}{d t}=-\frac{\partial H}{\partial P}, & \lambda_{P}\left(T_{f}\right)=0, \\
\frac{d \lambda_{E}}{d t}=-\frac{\partial H}{\partial E}, & \lambda_{E}\left(T_{f}\right)=0, & \frac{d \lambda_{T}}{d t}=-\frac{\partial H}{\partial T}, & \lambda_{T}\left(T_{f}\right)=0, \\
\frac{d \lambda_{I}}{d t}=-\frac{\partial H}{\partial I}, & \lambda_{I}\left(T_{f}\right)=0, & \frac{d \lambda_{A}}{d t}=-\frac{\partial H}{\partial A}, & \lambda_{A}\left(T_{f}\right)=0,
\end{aligned}
$$

Furthermore, by differentiating the Hamiltonian $H$ with respect to $u_{1}, u_{2}$, and $u_{3}$ on $U$, 
respectively,

$$
\begin{array}{ll}
\frac{\partial H}{\partial u_{1}}=C_{1} u_{1}-\left(\lambda_{S}-\lambda_{E}\right) S=0 & \text { at } u_{1}=u_{1}^{*} \\
\frac{\partial H}{\partial u_{2}}=C_{2} u_{2}-\left(\lambda_{P_{1}}-\lambda_{T} P=0\right. & \text { at } u_{2}=u_{2}^{*}, \\
\frac{\partial H}{\partial u_{3}}=C_{3} u_{3}-\left(\lambda_{A}-\lambda_{T}\right) A=0 & \text { at } u_{3}=u_{3}^{*} .
\end{array}
$$

Hence, solving for $u_{1}^{*}, u_{2}^{*}$ and $u_{3}^{*}$ on the interior sets gives

$$
\begin{aligned}
& u_{1}^{*}=\frac{\left(\lambda_{S}-\lambda_{E}\right) S^{*}}{C_{1}}, \\
& u_{2}^{*}=\frac{\left(\lambda_{P}-\lambda_{T}\right) P^{*}}{C_{2}}, \\
& u_{3}^{*}=\frac{\left(\lambda_{A}-\lambda_{T}\right) A^{*}}{C_{3}} .
\end{aligned}
$$

Now, let us consider the control bound, $0 \leq u_{i}^{*} \leq 1$ for $i=1,2$, 3. By using the bounds for control $u_{1}^{*}$, we get the following solution:

$$
u_{1}^{*}=\left\{\begin{array}{cl}
0 & \text { if } \frac{\left(\lambda_{\mathrm{S}}-\lambda_{\mathrm{E}}\right) S^{*}}{C_{1}} \leq 0 \\
\frac{\left(\lambda_{\mathrm{S}}-\lambda_{\mathrm{E}}\right) S^{*}}{C_{1}} & \text { if } 0<\frac{\left(\lambda_{\mathrm{S}}-\lambda_{\mathrm{E}}\right) S^{*}}{C_{1}}<1 \\
1 & \text { if } \frac{\left(\lambda_{\mathrm{S}}-\lambda_{\mathrm{E}}\right) S^{*}}{C_{1}} \geq 1 .
\end{array}\right.
$$

The compact representation of the control $u_{1}^{*}$ is

$$
u_{1}^{*}=\min \left\{\max \left(0, \frac{\left(\lambda_{S}-\lambda_{E}\right) S^{*}}{C_{1}}\right), 1\right\} .
$$

Similarly, controls $u_{2}^{*}$ and $u_{3}^{*}$ can be obtained in the same way and hence are written as

$$
\begin{aligned}
& u_{2}^{*}=\min \left\{\max \left(0, \frac{\left(\lambda_{P}-\lambda_{T}\right) P^{*}}{C_{2}}\right), 1\right\}, \\
& u_{3}^{*}=\min \left\{\max \left(0, \frac{\left(\lambda_{A}-\lambda_{T}\right) A^{*}}{C_{3}}\right), 1\right\} .
\end{aligned}
$$

Thus, we get the characterization of the optimal control as in Equation (9).

\section{Numerical Simulations and Cost-effectiveness Analysis}

In this section, we study numerically the effect of optimal control strategies such as educational campaigns, therapy on pre-AIDS, and therapy on full-blown AIDS using parameter values given by a study [8] as the following:

$$
\begin{aligned}
& \Lambda=33.638, \beta_{1}=0.1422, \beta_{2}=0.711, \alpha_{1}=0.0909, \alpha_{2}=0.0667, \delta=0.3 \\
& \sigma_{1}=0.198, \sigma_{2}=0.4621, \text { and } \mu=0.0139
\end{aligned}
$$


The optimal control set is obtained by completing the optimality system, which consists of the state system (1) and the adjoint system (8) through the forward-backward Sweep method [16]. We start to solve system (1) with a guess for the controls forward in time using the fourth-order Runge-Kutta scheme. Based on the transversality condition (7) as initial values, the adjoint system (8) is solved backward in time using the fourth-order Runge-Kutta scheme and the current solution iteration of the system. Then, the controls are updated using a convex combination of the previous control values and the new control values from (9). This process is repeated and iterations are stopped if the values of the state equation at the present is very close to the previous iteration values. We describe the controls in the following strategies using parameter values $(10)$ and the final time $T_{\mathrm{f}}=10$; the values of the weight function are taken as

$$
w_{1}=20, w_{2}=75, w_{3}=85,
$$

and the initial state of the variables are

$$
S(0)=957263, E(0)=959, I(0)=67, P(0)=34, T(0)=996, A(0)=89 .
$$

Furthermore, we investigate and compare the numerical results of the effects of different optimal control strategies on the spread of HIV in a population; we will consider the following combinations of educational campaigns, therapy on pre-AIDS, and therapy on full-blown AIDS with seven strategies:

Strategy 1: control of educational campaigns,

Strategy 2: control of therapy on pre-AIDS,

Strategy 3: control of therapy on full-blown AIDS,

Strategy 4: control of educational campaigns and therapy on pre-AIDS,

Strategy 5: control of educational campaigns and therapy on full-blown AIDS,

Strategy 6: control of therapy on pre-AIDS and therapy on full-blown AIDS,

Strategy 7: control of educational campaigns, therapy on pre-AIDS, and therapy on full-blown AIDS.

\subsection{Strategy 1: Control of Educational Campaigns}

In Strategy 1, control of educational campaigns $\left(u_{1}\right)$ is used to optimize the objective function $J$. Figures 1(a)-(c) show that the control of educational campaigns resulted in the graph of asymptomatic infection $(I)$, pre-AIDS infection $(P)$, and full-blown $\operatorname{AIDS}(A)$ coinciding between cases with control and cases without control. This means that the control of educational campaigns has no effect on decreasing the number of asymptomatic infected, preAIDS infected and full-blown AIDS cases. The control profile of the control of educational campaigns is at the lower bound from the beginning to the end of the period, except at $t=9.99$ years, where the control $u_{1}$ is at $2.28 \times 10^{-4}$. (Figure $1(\mathrm{~d})$ ). 


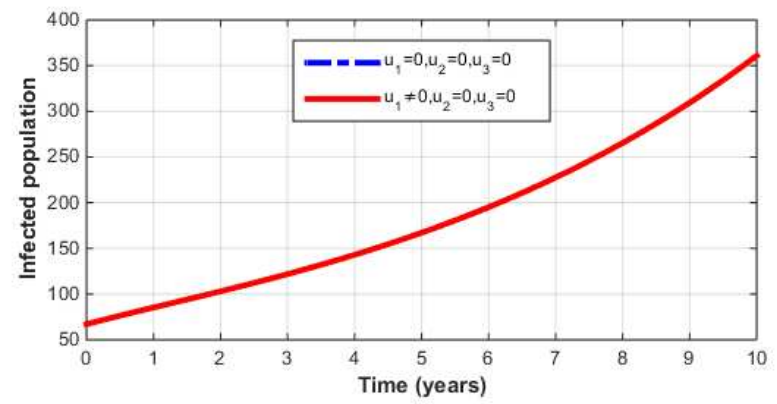

(a)

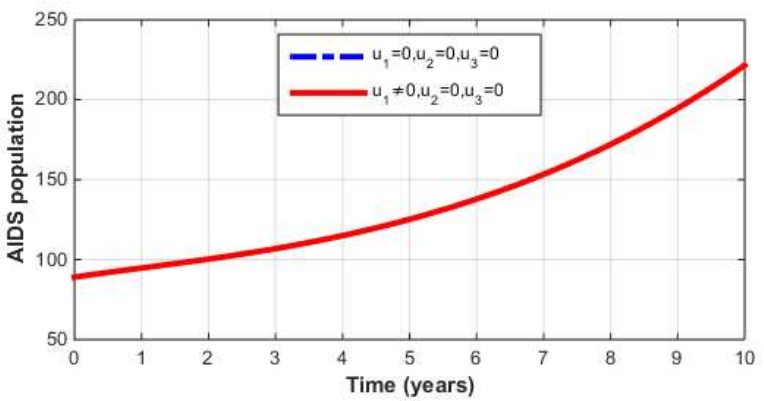

(c)

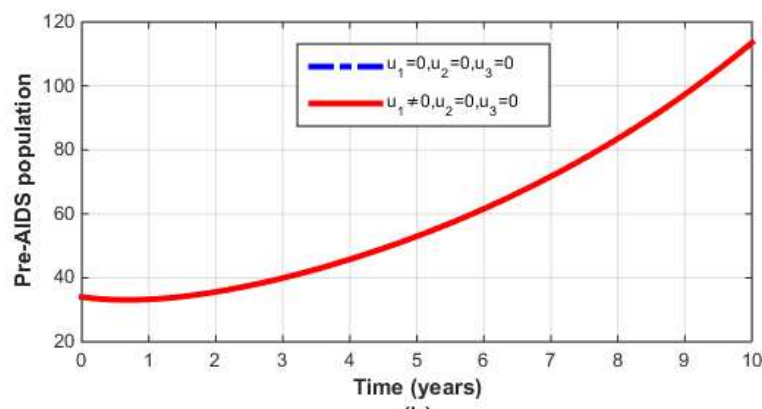

(b)

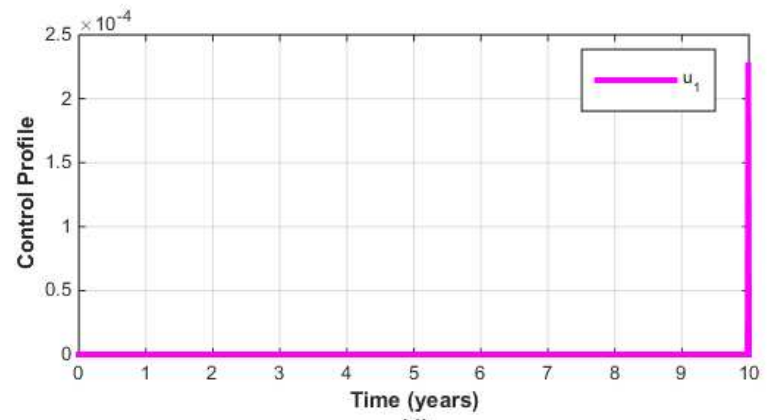

(d)

Figure 1: : Effect of the Control of Educational Campaigns $\left(u_{1}\right)$ on the Spread of Infection

\subsection{Strategy 2: Control of Therapy on Pre-AIDS}

When only control of antiretroviral therapy on pre-AIDS individuals is applied while other controls are set to zero, the significant effect occurs on the class of asymptomatic infected, pre-AIDS, and full-blown AIDS individuals (Figure 2(a)-(c)). The control profile of $u_{2}$ is at the maximum level for 2.88 years and declines gradually towards zero at the end of the period (Figure 2(d)).

\subsection{Strategy 3: Control of Therapy on Full-blown AIDS}

It can be seen that the control of antiretroviral therapy on full-blown AIDS $\left(u_{3}\right)$ used to optimize the objective function resulted in a significant drop in the number of full-blown AIDS individuals compared to without controls, while the graph of asymptomatic infected $(I)$ and infected preAIDS $(P)$ coincide between cases with control and cases without control (Figure 3(b)-(c)). This means that Strategy 3 has no effect on decreasing the number of asymptomatic infected and pre-AIDS individuals. Figure 3(d) shows that the control profile of therapy on full-blown AIDS $\left(u_{3}\right)$ is at the upper limit for 0.28 years before slowly decreasing, then slowly increasing to timet $=7.55$ years and dropping sharply towards zero at the end.

\subsection{Strategy 4: Control of Educational Campaigns and Therapy on Pre-AIDS}

The combination of control of educational campaigns and therapy on pre-AIDS produced the same resultsas Strategy 2 in decreasing the number of asymptomatic infected, pre-AIDS, and full-blown AIDS individuals compared to without controls (Figure 4(a)-(c)). Figure 4(d) shows 


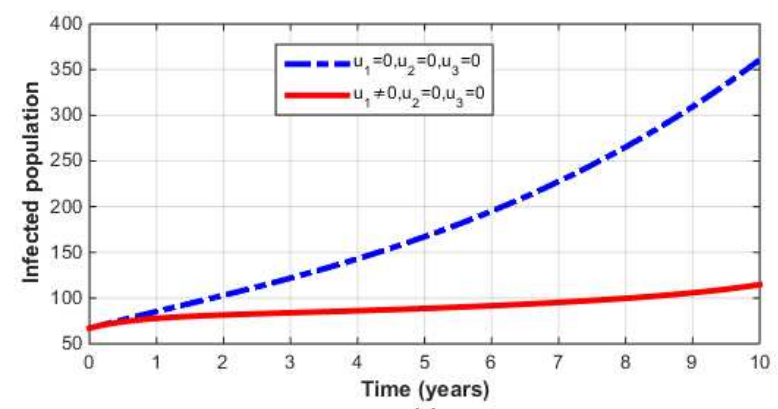

(a)

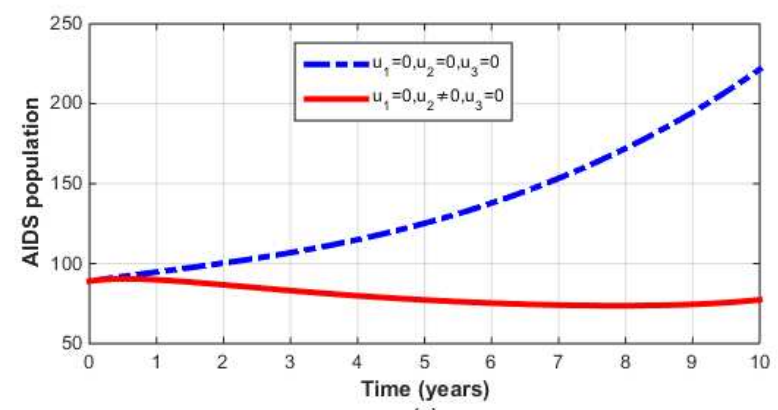

(c)

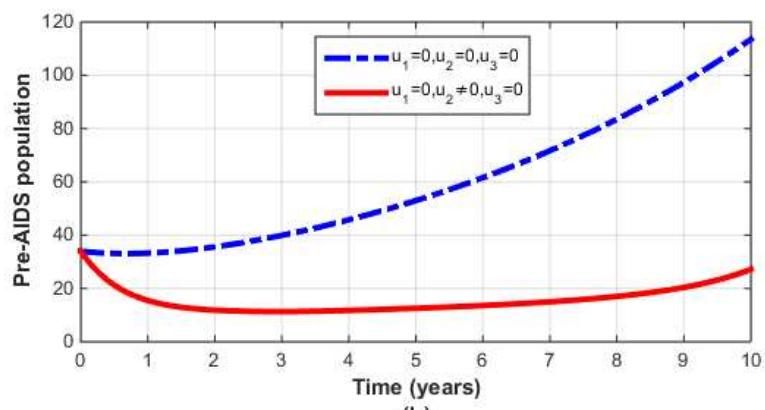

(b)

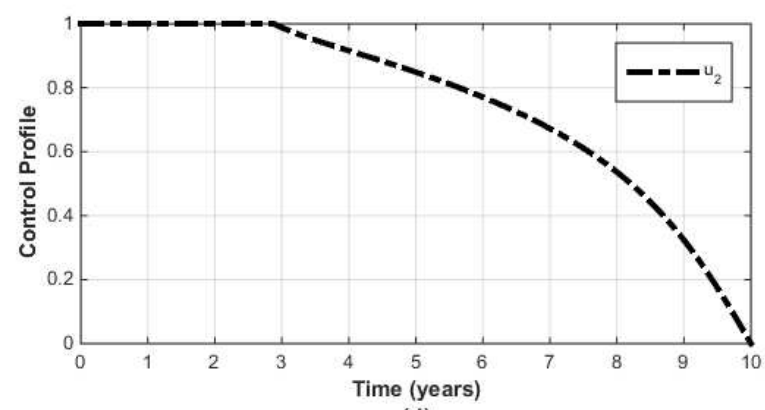

(d)

Figure 2: Effect of Control of Therapy on Pre-AIDS $\left(u_{2}\right)$ on the Spread of Infection

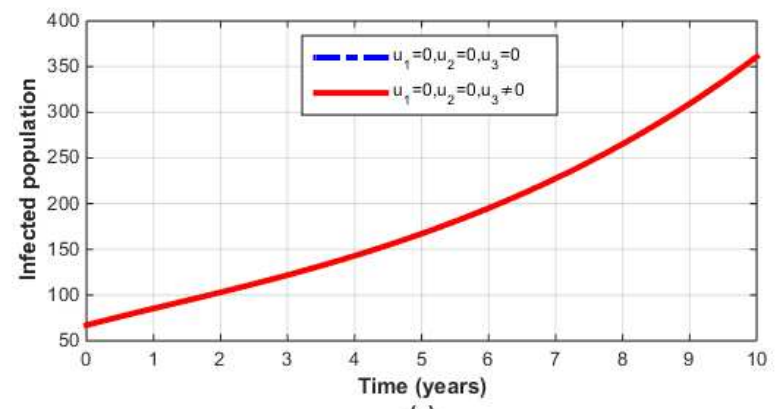

(a)

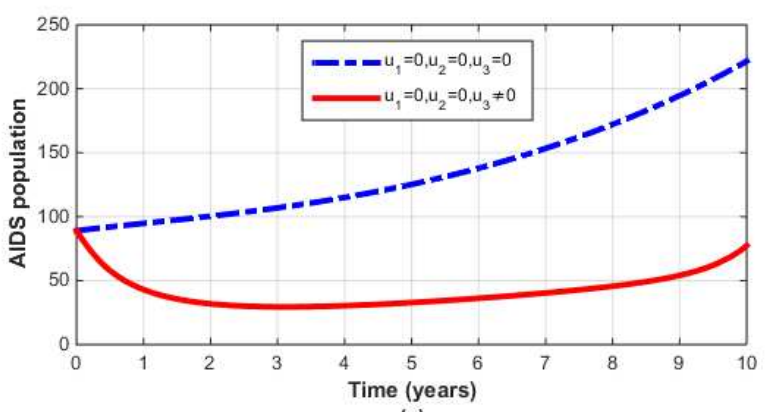

(c)

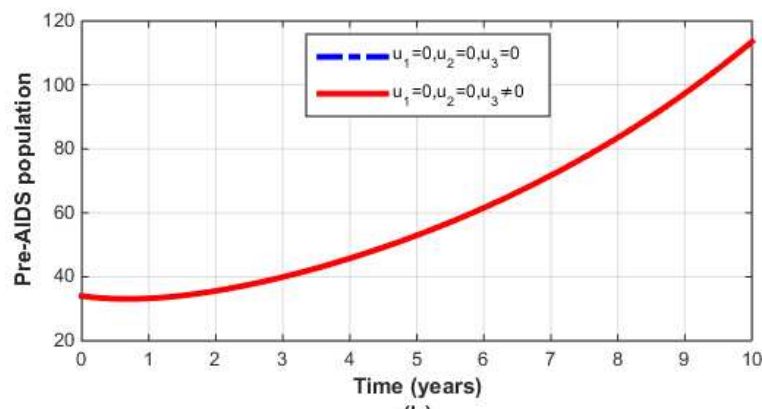

(b)

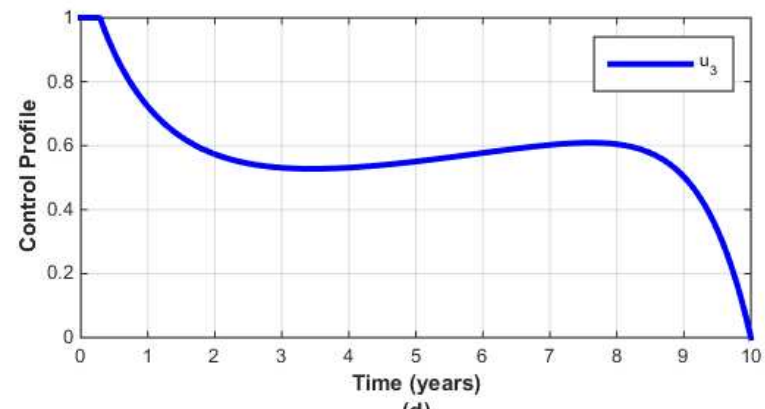

(d)

Figure 3: Effect of Control of Therapy on Full-blown AIDS $\left(u_{3}\right)$ on the Spread of Infection 
that the control profile of therapy on pre-AIDS is also the same as in Strategy 2, while the control profile of the control of educational campaigns is at the lower limit within the period of time.

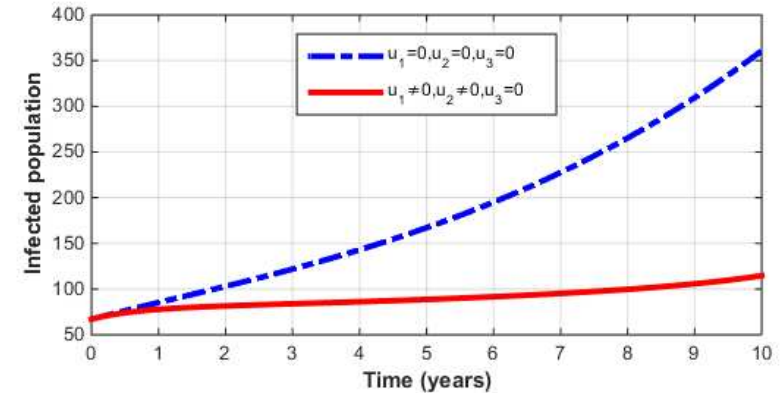

(a)

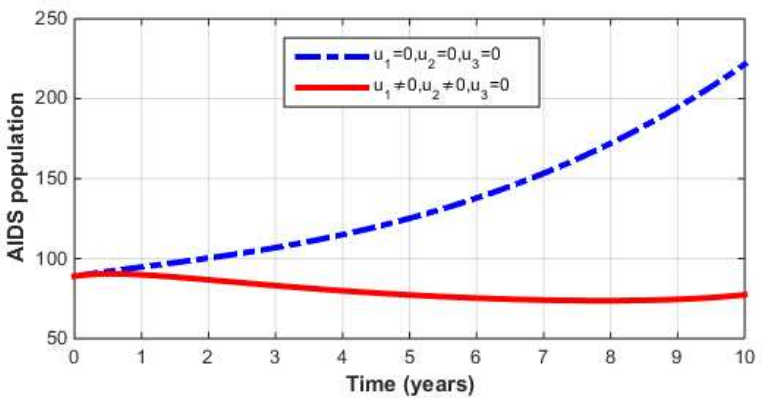

(c)

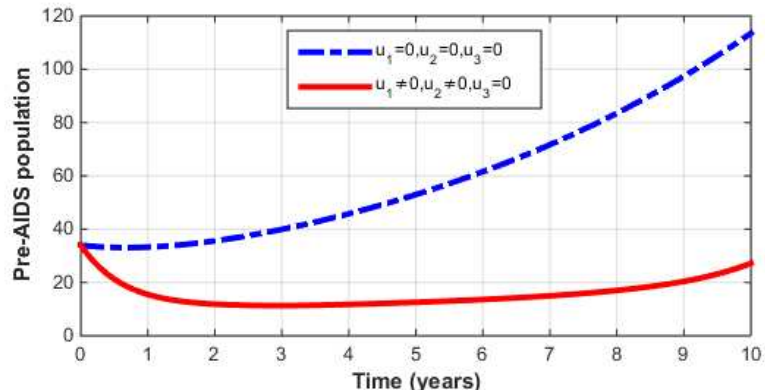

(b)

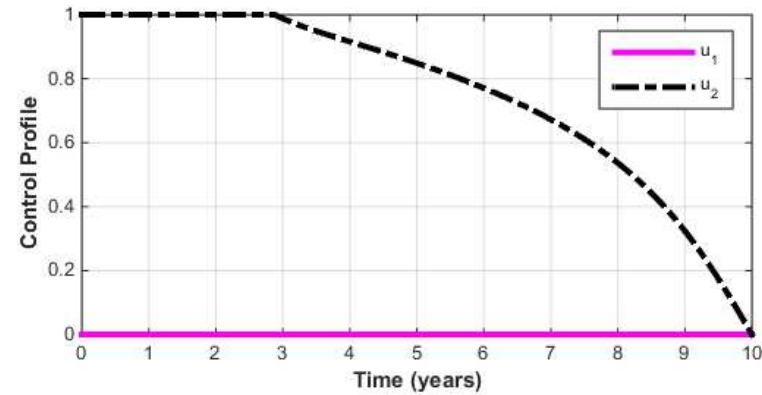

(d)

Figure 4: Effect of Control on Educational Campaigns $\left(u_{1}\right)$ and Therapy on Pre-AIDS $\left(u_{2}\right)$ on the Spread of Infection

\subsection{Strategy 5: Control of Educational Campaigns and Therapy on Full-blown AIDS}

Figure 5(a)-(c) shows that the combination of control of educational campaigns and control of therapy on full-blown AIDS produced the same results as Strategy 3 in decreasing the number of asymptomatic infected, pre-AIDS infected, and full-blown AIDS individuals compared to without controls. The control profile of therapy on pre-AIDS in Strategy 5 is also the same as in Strategy 2 (Figure 5(d)) and the control profile of the control of educational campaigns is at the lower limit within the period of time.

\subsection{Strategy 6: Control of Therapy on Pre-AIDS and Therapy on Full-blown AIDS}

In Figure 6 (a)-(c), it can be seen that the control strategy combination of therapy on pre-AIDS and therapy on full-blown AIDS resulted in a significant drop in the number of asymptomatic infected, pre-AIDS, and full-blown AIDS individuals compared to without controls. The control profile of antiretroviral therapy on pre-AIDS $\left(u_{2}\right)$ is at the upper limit for 2 years before decreasing gradually towards the lower limit at the end. The control profile of antiretroviral 


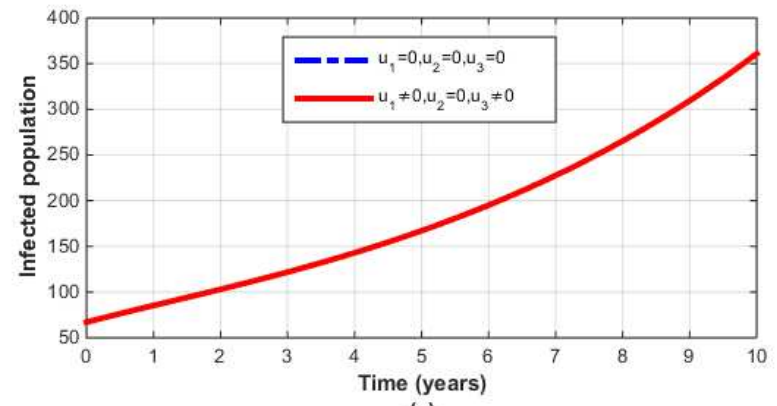

(a)

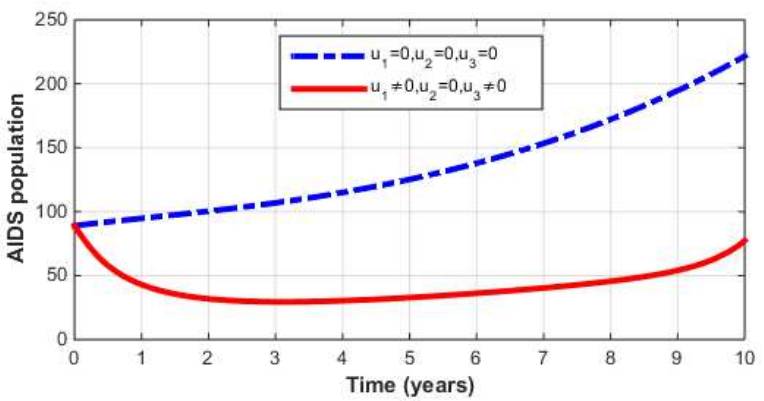

(c)

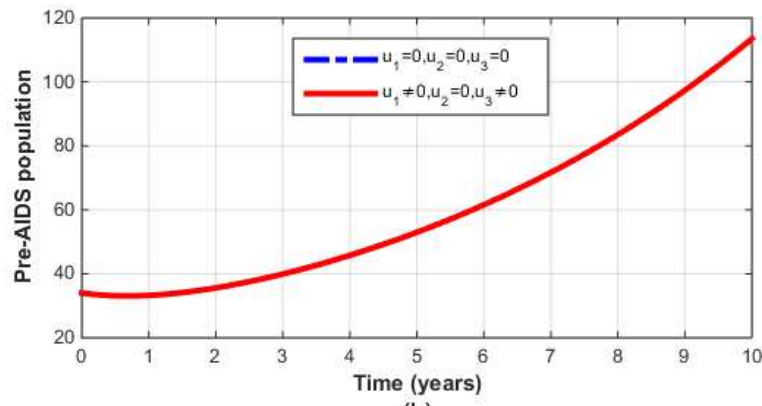

(b)

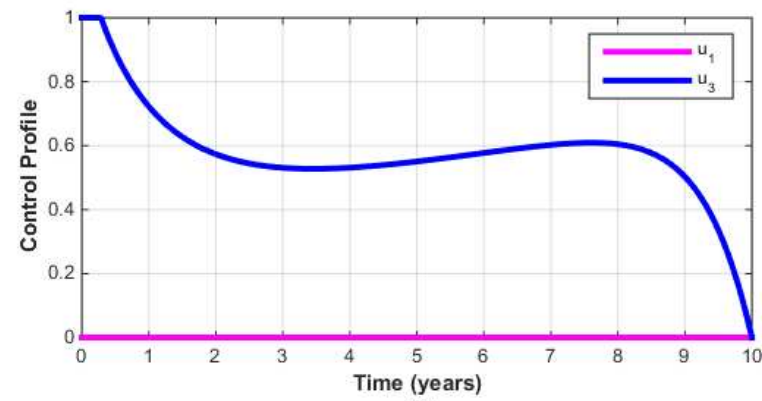

(d)

Figure 5: Effect of Control of Educational Campaigns $\left(u_{1}\right)$ and Therapy on Full-blown AIDS $\left(u_{3}\right)$ on the Spread of Infection

therapy on full-blown AIDS $\left(u_{3}\right)$ is at the upper limit for 0.38 years before decreasing periodically to the lower limit at the end (Figure $6(\mathrm{~d})$ ).

\subsection{Strategy 7: Control of Educational Campaigns, Therapy on Pre-AIDS, and Therapy on Full-blown AIDS}

Figure 7(a)-(c) shows that the combination of all three controls (educational campaigns, therapy on pre-AIDS, and therapy on full-blown AIDS) produced the same result as Strategy 6 in decreasing the number of asymptomatic infected, pre-AIDS infected, and full-blown AIDS individuals compared to without controls. The control profile of therapy on pre-AIDS and therapy on full-blown AIDS in Strategy 7 is also the same as in Strategy 6, while the control profile of the control of educational campaigns is at the lower limit within the period of time (Figure 5(d)).

\subsection{Cost-Effectiveness Analysis}

In this section, we focus on comparing the four control strategies in Section 4.1-4.4 to determine the most cost-effective strategy using cost-effectiveness analysis. To perform cost-effectiveness analysis, we follow the method as applied in several studies [11, 17, 19]. To achieve this, we evaluate the costs using the incremental cost-effectiveness ratio ICER to compare the differences between the various costs and health outcomes of the two competing intervention strategies. 


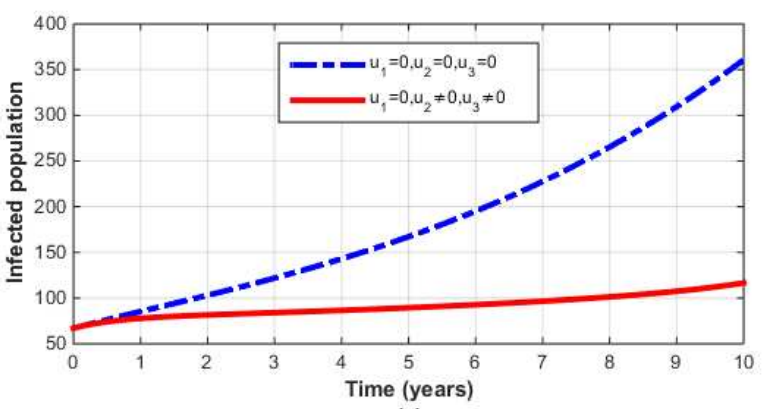

(a)

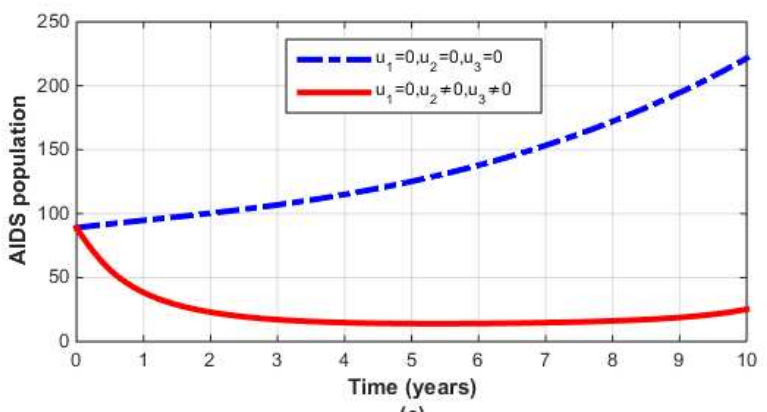

(c)

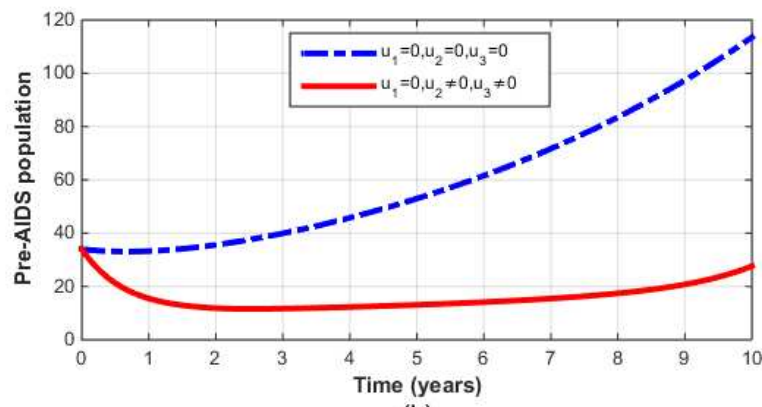

(b)

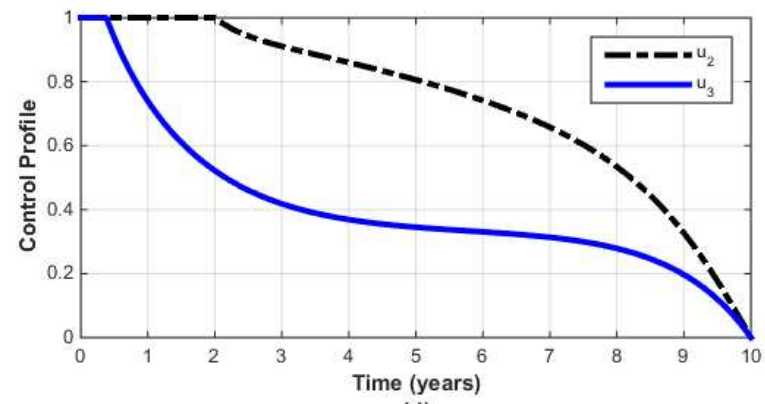

(d)

Figure 6: Effect of Control of Therapy on Pre-AIDS $\left(\mathrm{u}_{2}\right)$ and Therapy on Full-blown AIDS $\left(u_{3}\right)$ on the Spread of Infection

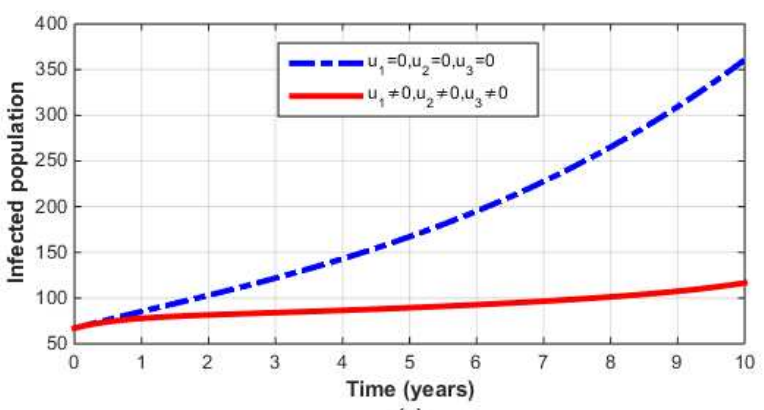

(a)

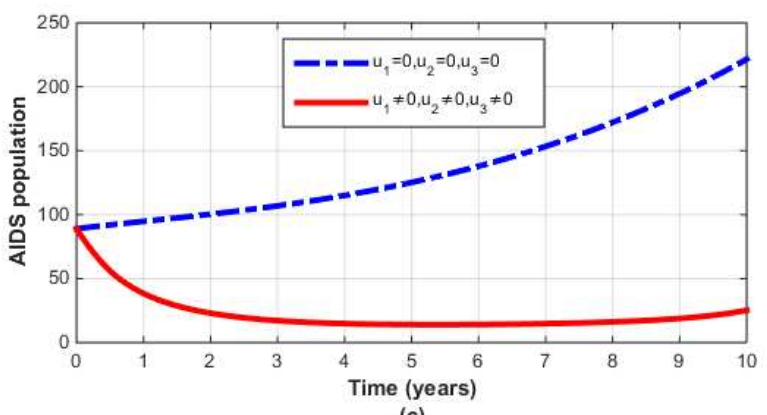

(c)

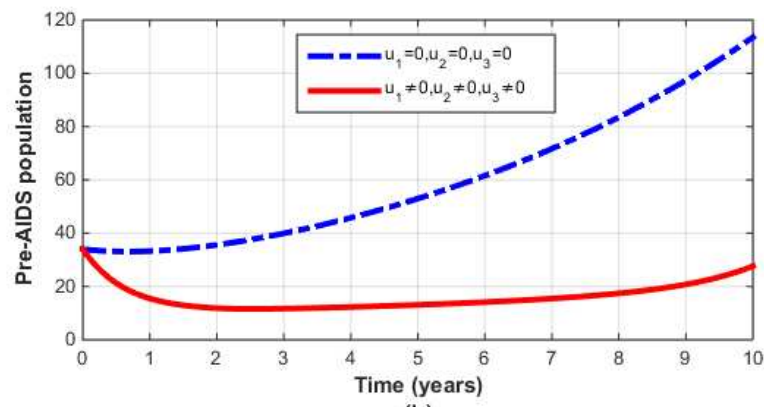

(b)

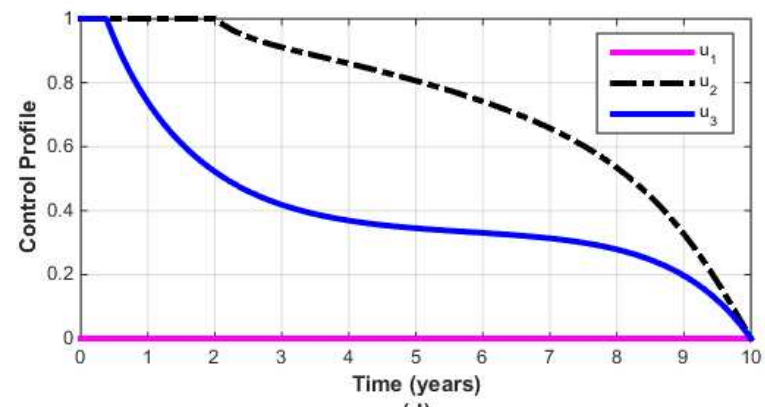

(d)

Figure 7: Effect of Control of Educational Campaigns $\left(u_{1}\right)$, Therapy on Pre-AIDS $\left(u_{2}\right)$, and Therapy on Full-blown AIDS $\left(u_{3}\right)$ on the Spread of Infection. 
The ICER is defined by:

$$
\mathrm{ICER}=\frac{\text { Defference in costs in strategies } \mathrm{i} \text { and } \mathrm{j}}{\text { Defference in infected averted in strategies } i \text { and } \mathrm{j}} .
$$

The ICER numerator includes differences in intervention costs, averted disease costs, and costs of prevented cases, as well as averted productivity losses, if applicable. The ICER denominator is the difference in health outcomes (for example the total number of infections averted andthe number of susceptibility cases prevented).

Given two competing Strategies $P$ and $Q$, where Strategy $Q$ has higher effectiveness than Strategy $P$, the ICER values are calculated with the below equations.

$$
\begin{aligned}
\operatorname{ICER}(P) & =\frac{T C(P)}{T A(P)}, \\
\operatorname{ICER}(Q) & =\frac{T C(Q)-T C(P)}{T A(Q)-T A(P)} .
\end{aligned}
$$

In this paper, the total costs $(T C)$ and the total cases averted $(T A)$ as implemented during the given period for strategy $i$ for $i=1,2,3,4$ are

$$
\begin{aligned}
& T C(i)=\int_{0}^{T_{f}}\left(C_{1} u_{1}^{*}(t) S^{*}(t)+C_{2} u_{2}^{*}(t) P^{*}(t)+C_{3} u_{3}^{*}(t) A^{*}(t)\right) d t \\
& T A(i)=\int_{0}^{T_{f}}\left[(I(t)+P(t)+A(t))-\left(I^{*}(t)+P^{*}(t)+A^{*}(t)\right)\right] d t
\end{aligned}
$$

where $C_{\mathrm{i}}$ corresponds to the person unit cost of the three possible interventions: control of educational campaigns for condom use $\left(C_{1}\right)$, antiretroviral therapy for pre-AIDS individuals $\left(C_{2}\right)$, and antiretroviral therapy for full-blown AIDS individuals $\left(C_{3}\right)$, while $\left(I^{*}(t), P^{*}(t), A^{*}(t)\right)$ is the optimal solution associated to the optimal control $\left(u_{1}^{*}, u_{2}^{*}, u_{3}^{*}\right)$.

Next, we simulate the model using seven intervention strategies. Using these simulation results, the control strategies are ranked in order of increased numbers of averted infections. The difference between the total infected individuals without control and the total infected individuals with controls are used to determine the "total infection averted" used in the costeffectiveness analysis table.

The total cost generated by the control strategy is proportional to the number of controls used. Table 1 presents control strategies ranked in ascending order according to the total averted infections.

Strategy 1 is compared with Strategy 3 with respect to increased effectiveness, in reference to Table 1. Using Equation (13), the ICER values are calculated below:

$$
\begin{aligned}
& \operatorname{ICER}(1)=\frac{1.0437 \times 10^{-7}}{2.2574 \times 10^{-9}}=4.6235 . \\
& \operatorname{ICER}(3)=\frac{301.513-1.0437 \times 10^{-7}}{937.1-2.2574 \times 10^{-9}}=0.3218 . \\
& \operatorname{ICER}(5)=\operatorname{ICER}(3) .
\end{aligned}
$$

The comparison between Strategy 1 and Strategy 3 indicates that $I C E R(3)<I C E R(1)$. This means that Strategy 1 is dominated by Strategy 3. Similarly, Strategy 1is dominated by 
Table 1: Total Costs for Strategies 1-7 in Increasing Order of Total Averted Infections

\begin{tabular}{|c|c|c|}
\hline Strategy & Total averted infections $(T A)$ & Total cost $(T C)$ \\
\hline 1 & $2.2574 \times 10^{-9}$ & $21.0437 \times 10^{-8}$ \\
\hline 3 & 937.1 & 301.513 \\
\hline 5 & 937.1 & 301.513 \\
\hline 2 & 1920.8 & 485.218 \\
\hline 4 & 1920.8 & 485.218 \\
\hline 6 & 2486.7 & 633.859 \\
\hline 7 & 2486.7 & 633.859 \\
\hline
\end{tabular}

Strategy 5. Hence, Strategy 1 is less effective than Strategies 3 and 5. Therefore, Strategy 1 is excluded from the set of alternatives.

Next, Strategies 3 and 5 are compared with Strategy 2. The ICER values for Strategy 3 and Strategy 2 are calculated below:

$$
\begin{aligned}
& \operatorname{ICER}(3)=\frac{301.513}{937.1}=0.3218 . \\
& \operatorname{ICER}(5)=\operatorname{ICER}(3) . \\
& \operatorname{ICER}(2)=\frac{485.218-301.513}{1920.8-937.1}=0.1867 . \\
& \operatorname{ICER}(4)=\operatorname{ICER}(2) .
\end{aligned}
$$

The comparison of ICER (5) and ICER (2) reveals a cost savings of 0.1867 for Strategy 2 over Strategies 3 and 5. The smaller ICER (2) for Strategy 2 implies that Strategies 3 and 5 are dominated by Strategy 2. Similarly, Strategies 3 and 5 are dominated by Strategy 4. This means that Strategies 3 and 5 are more expensive and less effective than Strategies 2 and 4 . Therefore, Strategies 3 and 5 are excluded from the set of alternatives.

Finally, Strategies 2 and 4 are compared with Strategy 6. The ICER values for Strategy 2 and Strategy 6 are calculated below:

$$
\begin{aligned}
& \operatorname{ICER}(2)=\frac{485.218}{1920.8}=0.2526 . \\
& \operatorname{ICER}(4)=\operatorname{ICER}(2) \\
& \operatorname{ICER}(6)=\frac{633.859-485.218}{2486.7-1920.8}=0.262 . \\
& \operatorname{ICER}(7)=\operatorname{ICER}(6) .
\end{aligned}
$$

The comparison of Strategies 2 and 4 with Strategy 3 indicates that Strategy 6 is more costly and less effective than Strategy 2, as $\operatorname{ICER}(2)<\operatorname{ICER}(6)$. Hence, Strategy 6 is dominated by Strategy 4, as $\operatorname{ICER}(4)=\operatorname{ICER}(2)$. Similarly, Strategy 7 is dominated by Strategies 2 and 4. This means that Strategies 6 and 7 are more expensive and less effective than Strategies 2 and 4. Therefore, Strategies 6 and 7 are excluded from the set of alternatives. Thus, the conclusion is that Strategy 2 (control of therapy on pre-AIDS)and Strategy 4 (the combination of the control of educational campaigns and therapy on pre-AIDS) are the most effective strategies. 


\section{Conclusion}

In this paper, we performed an optimal control analysis for the HIV model to investigate the effect of educational campaigns, antiretroviral therapy on pre-AIDS, and antiretroviral therapy in full-blown AIDS on HIV dynamics. Pontryagin's Maximum Principle was used to derive and analyze the necessary conditions for optimal control strategies: condom campaigns $\left(u_{1}\right)$, antiretroviral therapy on pre-AIDS $\left(u_{2}\right)$ and antiretroviral therapy on full-blown AIDS $\left(u_{3}\right)$ to minimize the spread of HIV.

Numerically, the optimal strategies that include antiretroviral therapy on pre-AIDS (Strategies 2, 4, 6 and 7) showed significant differences in the number of asymptomatic infected, pre-AIDS infected, and full-blown AIDS individuals compared to without controls. The optimal strategies that include antiretroviral therapy on full-blown AIDS (Strategy 3 and 5) only have positive effects in decreasing the number of full-blown AIDS individuals and have no positive impact on asymptomatic infected and pre-AIDS class individuals. The control profile of the control of educational campaigns is at the lower limit until the end of the period and does not have a positive effect on reducing the number of infected individuals. In the case of limited resources, Strategy 2 (control of therapy on pre-AIDS) and Strategy 4 (the combination of control of educational campaigns and antiretroviral therapy on pre-AIDS) are the optimal and most effective strategies.

\section{Acknowledgments}

This work was partially supported by the DRPM RISTEKDIKTI, Directorate-General of Research and Development Reinforcement, and Ministry of Research, Technology, and Higher Education of Indonesia in accordance with the Letter of Appointment for the Agreement of Implementation of Research Program, Number054/SP2H/LT/DRPM/2018.

\section{References}

[1] Anderson, R. M. The role of mathematical models in the study of HIV transmission and the epidemiology of AIDS. J. AIDS. 2001. 1: 214-256.

[2] Brauer, F. and Castillo-Chavez, C. Mathematical Models in Population Biology and Epidemiology. Text in Applied Mathematics, 40, New York: Springer-Verlag. 2001.

[3] Tripathi, A., Naresh, R. and Sharma, D. Modelling the effect of screening of unaware infectives on the spread of HIV infection. Appl. Math. Comput. 2007. 184: 1053-1068.

[4] Mukandavire, Z., Garira, W. and Tchuenche, J. M. Modelling effects of public health educational campaigns on HIV/AIDS Transmission Dynamics. Applied Mathematical Modelling. 2009. 33 (4): 2084-2095.

[5] Joshi, H. R., Lenhart, S., Albright, K. and Gipson, K. Modeling the effect of information campaigns on the HIV epidemic in Uganda, Mathematical Biosciences and Engineerings, 2008. 5: 757-770.

[6] Marsudi, Hidayat, N. and Wibowo, R. B. E. Optimal control for controlling the spread of HIV dynamics with educational campaigns and antiretroviral therapy. IOP Conf. Series: Journal of Physics: Conf. Series. 2018. 1028: 012115. 
[7] Cuslaw, R. V. Optimal HIV treatment by maximizing immune response. Journal Math Biol. 2004. 48: 545-562.

[8] Marsudi, Marjono, and Andari, A. Modeling the effect of screening and therapy on the dynamics of HIV in Malang, Proceeding The $5^{\text {th }}$ Annual Basic Science International Conference. 2015. 5: $310-313$.

[9] Marsudi, Hidayat, N. and Wibowo, R. B. E. Sensitivity analysis of the parameters of an HIV/AIDS model with condom campaign and antiretroviral therapy, AIP Conference Proceedings. 2017. 1913: 020019.

[10] Naresh, R., Tripathi, A. and Sharma, D. Modelling the effect of risky sexual behavior on the spread of HIV/AIDS. International Journal of Applied Mathematics and Computation. 2009. 1 (3): 132-147.

[11] Agusto, F. B. and Elmojtaba, I. M. Optimal control and cost-effective analysis of malaria/visceral leishmaniasis co-infection. PloS ONE 2017. 12(2): e0171102.

[12] Blayneh, K., Cao Y. and Kwon, H-D. Optimal control of vector-borne diseases: treatment and prevention. Discrete Continues Dyn. Syst. Series B. 2009. 11 (3): 587-611.

[13] Fleming, W. H. and Rishel, R. W. Deterministic and Stochastic Optimal Control, New York: Springer Verlag. 1975.

[14] Hota, S., Agusto, F., Joshi, H. R. and Lenhart, S. Optimal control and stability analysis of an epidemic model with education campaign and treatment. Dynamical Systems, Differential Equations, and Applications, AIMS Proceedings. 2015. 621-634.

[15] Kirschner, D., Lenhart, S. and Serbin, S. Optimal control of the Chemotherapy of HIV. Journal Math Biol. 1997. 35: 775-792.

[16] Lenhart, S. and Workman, J. T., Optimal Control Applied to Biological Models. London: Chapman and Hall. 2007.

[17] Okosun, K. O., Makinde, O. D. and Takaidza, I. Impact of optimal control on the treatment of HIV/AIDS and screening of unaware infectives. Applied Mathematical Modelling. 2013. 37: $3802-3820$.

[18] Tchuenche, J. M., Khamis, S. A., and Agusto, F. B., Optimal control and sensitivity analysis of an influenza model with treatment and vaccination, Acta Biotheor. 2011. 59: $1-28$.

[19] Okosun, K. O., Rachid, O. and Marcus, N. Optimal control strategies and cost-effectiveness analysis of a Malaria model. Bio Systems. 2013.111: 83-101.

[20] Pontryagin, L. S., Boltyanskii, V. G., Gamkrelidze, R. V. and Mishchenko, E. F. The Mathematical Theory of Optimal Processes. New York: Wiley. 1962. 